\title{
Integrin alpha-2 Inhibitor E7820
}

National Cancer Institute

\section{Source}

National Cancer Institute. Integrin alpha-2 Inhibitor E7820. NCI Thesaurus. Code C61501.

A small molecule and aromatic sulfonamide derivative with potential antiangiogenic and antitumor activities. E7820 inhibits angiog enesis by suppressing integrin alpha 2, a cell adhesion molecule expressed on endothelial cells. Inhibition of integ rin alpha 2 leads to an inhibition of cell-cell interactions, endothelial cell-matrix interactions, vascular endothelial cell proliferation and angiogenesis. 